\title{
Feasibility of a clinical trial to assess the effect of dietary calcium $v$. supplemental calcium on vascular and bone markers in healthy postmenopausal women
}

\author{
Angel M. Ong ${ }^{1,2}$, Hope A. Weiler ${ }^{2}$, Michelle Wall ${ }^{1}$, Rouba Haddad ${ }^{3}$, Jessica Gorgui ${ }^{1}$, \\ Stella S. Daskalopoulou ${ }^{1,4}$, David Goltzman ${ }^{1,4}$ and Suzanne N. Morin ${ }^{1,4_{*}}$ \\ ${ }^{1}$ Research Institute, McGill University Health Centre, Montreal, QC, Canada, H3G $1 A 4$ \\ ${ }^{2}$ School of Dietetics and Human Nutrition, McGill University, Ste-Anne-de-Bellevue, QC, Canada, H9X 3 V9 \\ ${ }^{3}$ Department of Nutrition, Shriners Children's Hospital, Montreal, QC, Canada, H3G $1 A 6$ \\ ${ }^{4}$ Department of Medicine, McGill University, Montreal, QC, Canada, H4A 3J1 \\ (Submitted 10 November 2015 - Final revision received 11 February 2016 - Accepted 28 March 2016 - First published online 16 May 2016)
}

\section{Abstract}

Whether supplemental Ca has similar effects to dietary Ca on vascular and bone markers is unknown. The present trial investigated the feasibility of applying dietary and supplemental interventions in a randomised-controlled trial (RCT) aiming to estimate the effect of supplemental Ca as compared with dietary $\mathrm{Ca}$ on vascular and bone markers in postmenopausal women. In total, thirteen participants were randomised to a Ca supplement group (CaSuppl) $\left(750 \mathrm{mg}\right.$ Ca from $\mathrm{CaCO}_{3}+450 \mathrm{mg}$ Ca from food $+20 \mu \mathrm{g}$ vitamin D supplement) or a Ca diet group (CaDiet) (1200 mg Ca from food $+10 \mu \mathrm{g}$ vitamin D supplement). Participants were instructed on Ca consumption targets at baseline. Monthly telephone follow-ups were conducted to assess adherence to interventions ( $\pm 20 \%$ of target total Ca) using the multiple-pass 24 -h recall method and reported pill count. Measurements of arterial stiffness, peripheral blood pressure and body composition were performed at baseline and after 6 and 12 months in all participants who completed the trial $(n 9)$. Blood and serum biomarkers were measured at baseline and at 12 months. Both groups were compliant to trial interventions ( $\pm 20 \%$ of target total Ca intake; pill count $\geq 80 \%$ ). CaSuppl participants maintained a significantly lower average dietary Ca intake compared with CaDiet participants throughout the trial (453 (sD 187) mg/d $v .1241$ (sD 319) mg/d; $P<0 \cdot 001$ ). There were no significant differences in selected vascular outcomes between intervention groups over time. Our pilot trial demonstrated the feasibility of conducting a large-scale RCT to estimate the differential effects of supplemental and dietary Ca on vascular and bone health markers in healthy postmenopausal women.

\section{Key words: Dietary interventions: Calcium: Arterial stiffness: Postmenopausal women}

Adequate intakes of $\mathrm{Ca}$ and vitamin $\mathrm{D}$ are essential for optimal bone health throughout adulthood to prevent osteoporosis and related fractures ${ }^{(1-6)}$. Current dietary reference intakes for Ca have been established by the Institute of Medicine for women over 50 years of age - that is, the Estimated Average Requirement (EAR), RDA and Tolerable Upper Intake Level (UL) are 1000, 1200 and $2000 \mathrm{mg} / \mathrm{d}$, respectively ${ }^{(7)}$. However, adequate intake of $\mathrm{Ca}$ can be difficult to achieve through dietary sources alone ${ }^{(8,9)}$.

According to the National Health and Nutrition Examination Survey 2003-2006 data, less than 10\% of American women over 50 years of age reached an intake of $1200 \mathrm{mg} / \mathrm{d}$ of Ca from food alone $^{(8)}$. Similarly, over $80 \%$ of Canadian women over 50 years of age had a Ca intake $<1000 \mathrm{mg} / \mathrm{d}$ from dietary sources according to the 2004 Canadian Community Health Survey ${ }^{(9)}$.
Thus, to ensure adequate total $\mathrm{Ca}$ intake for skeletal integrity, Ca supplements are widely recommended ${ }^{(10,11)}$. Collectively, data from North America documented that $49-67 \%$ of women aged $50-70$ years and $60-65 \%$ of those over 71 years of age reported using supplements that contain $\mathrm{Ca}^{(8,9)}$.

Ca supplementation is generally well tolerated but can be associated with mild gastrointestinal symptoms and with renal lithiasis ${ }^{(12)}$. More recently, concerns about the use of supplemental $\mathrm{Ca}$ have been expressed following the publication of two meta-analyses, which reported that Ca supplementation, with or without vitamin D, increased the risk of cardiovascular events, particularly myocardial infarctions ${ }^{(13,14)}$. However, other analyses in similar populations have not reported this adverse association between $\mathrm{Ca}$ supplementation and

Abbreviations: 25(OH)D, 25-hydroxy vitamin D; CaDiet, Ca diet group; cfPWV, carotid-femoral pulse wave velocity; crPWV, carotid-radial pulse wave velocity; CaSuppl, Ca supplement group; PTH, parathyroid hormone; PWV, pulse wave velocity.

* Corresponding author: Dr S. N. Morin, fax +1 514937 7298, email suzanne.morin@mcgill.ca 
cardiovascular events ${ }^{(15-18)}$. On the other hand, most studies demonstrated that dietary sources of Ca have not been linked with cardiovascular adverse events ${ }^{(13,19,20)}$ or have been shown to be favourable to cardiovascular health ${ }^{(21-24)}$. However, a recent report has raised uncertainty associating milk intake with a dose-dependent higher all-cause mortality rate, particularly cardiovascular death, in a large cohort of men and women over a 20 -year period $^{(25)}$.

CVD is a leading cause of morbidity and mortality globally in women after the age of 50 years, with coronary artery disease and stroke representing 23 and $18 \%$ of cause of death, respectively ${ }^{(26,27)}$. In practice, early damage to the vascular system can be assessed non-invasively and accurately by measuring arterial stiffness, a composite indicator of vascular health that is an independent predictor of CVD and cardiovascular events ${ }^{(28,29)}$. However, limited evidence exists on the effect of either supplemental or dietary Ca intake on arterial stiffness.

We conducted a 12-month pilot trial to determine the feasibility of controlled dietary and supplemental interventions of a randomised-controlled trial that aims to assess the effect of supplemental $\mathrm{Ca}$ as compared with dietary $\mathrm{Ca}$ on arterial stiffness and other vascular and bone markers in postmenopausal women. The primary objectives were to test the feasibility of nutritional counselling on adherence to the dietary and supplemental interventions, and the tolerability of $\mathrm{Ca}$ and vitamin D supplementation. The secondary exploratory objective was to contribute preliminary evidence on the effect of dietary $\mathrm{Ca} v$. supplemental $\mathrm{Ca}$ on vascular and bone health markers.

\section{Methods \\ Study design and population}

We conducted a randomised, single-blinded, parallel, 1-year intervention trial from May 2012 to January 2014. Healthy postmenopausal women aged 55 years and older ( $\geq 3$ years since last menstrual period) without any chronic disease were recruited through posters and local newspaper advertisements in Montreal, Quebec, and were screened for eligibility by phone. Exclusion criteria were established to avoid including women with clinical or subclinical vascular impairment due to cardiovascular risk factors or CVD. Specifically, postmenopausal women with lactose intolerance were excluded, as were those who smoked within the last 5 years, who had a $\mathrm{BMI}<20$ or $>30 \mathrm{~kg} / \mathrm{m}^{2}$, history of diabetes, pre-eclampsia, hypertension, atrial fibrillation or atherosclerosis. Participants who had used hormonal replacement therapy (excluding vaginal preparations) in the last 3 years, medications to treat hypertension or hypercholesterolaemia or medications known to affect bone metabolism (systemic glucocorticoids, bisphosphonates, receptor activator of $\mathrm{NF}-\kappa \mathrm{B}$ ligand inhibitor, selective oestrogen receptor modulators, calcitonin, teriparatide) within the past 12 months were also excluded. Those with a 10 -year absolute risk of major osteoporotic fractures $>20 \%$, computed using FRAX $^{\mathrm{TM}}$ without bone mineral density, were excluded as well ${ }^{(30)}$. All participants were required to refrain from using nutritional supplements for 2 months before study entry. The present trial was conducted according to the guidelines laid down in the Declaration of Helsinki, and ethics approval was granted by the McGill University Health Centre Research Ethics Board. Written informed consent was obtained from all participants (GEN-11-231).

On-site visits occurred at baseline and at 6 and 12 months after randomisation at the same research site. Participants were asked to abstain from vigorous physical activity and alcohol consumption for $48 \mathrm{~h}$ and to fast overnight for $12 \mathrm{~h}$ before their visit. Participants were randomised to one of two interventions on the day of the baseline visit. A web-based patient randomisation service (http://www.randomizer.at) was used to generate permuted block randomisation in six-block intervals. The interventions were as follows: Ca supplement group (CaSuppl), $750 \mathrm{mg} \mathrm{Ca}$ carbonate supplement (Euro-Cal; Euro-Pharm International Canada Inc.) $+450 \mathrm{mg}$ from food sources + vitamin D supplement of $20 \mu \mathrm{g}$ (800 IU) daily or Ca diet group (CaDiet), $1200 \mathrm{mg}$ of Ca from food sources + vitamin D supplement of $10 \mu \mathrm{g}$ (400 IU) daily. Although very few foods naturally contain vitamin D, the CaDiet supplies $10 \mu \mathrm{g}$ (400 IU) of dietary vitamin $\mathrm{D}$ from fluid milk and alternative plant-based beverages enriched with $\mathrm{Ca}$, which are fortified with vitamin $\mathrm{D}$ in Canada.

Participants randomised to the CaSuppl arm were instructed by the research dietitian to limit their daily intake of dairy products and alternative Ca-rich foods to one small portion (providing approximately $150 \mathrm{mg} /$ serving). Participants randomised to the CaDiet arm were instructed to increase their intake of dairy products and alternative Ca-rich foods to three portions a day (providing approximately $300 \mathrm{mg} /$ serving). A total daily intake of $1200 \mathrm{mg}$ of $\mathrm{Ca}$, which included $300 \mathrm{mg}$ of $\mathrm{Ca}$ from other foods in the diet, such as vegetables and grains ${ }^{(31)}$, was obtained from the combination of supplementation and dietary sources in the CaSuppl group, and through dietary sources alone in the CaDiet group. Instructions on how to estimate portion sizes were provided at the baseline visit by the dietitian using food models and common household items. Educational tools including the Calcium Calculator ${ }^{\mathrm{TM}}$ by the British Columbia Dairy Foundation ${ }^{(32)}$, the nutrition label reading handout by Health Canada ${ }^{(33)}$ and sample menus were provided. Participants received monthly follow-up telephone calls to monitor health status, adverse events and compliance to the assigned intervention.

\section{Measurements}

Measures of feasibility. The primary outcome measures for this pilot trial include participant adherence to the trial protocol and compliance to trial interventions, as well as tolerability of $\mathrm{Ca}$ and vitamin $\mathrm{D}$ supplementation. Compliance to trial interventions was defined as a mean total Ca intake $\pm 20 \%$ of target (i.e. $1200 \pm 240 \mathrm{mg}$ ), as per intervention assignment, and use of Ca supplements $\geq 80 \%$. Tolerability of supplements was assessed by reported adverse events, and acceptability of measurements was assessed by direct questioning by research personnel. 
Questionnaires. Past medical history and family history of CVD and bone disease were surveyed at the baseline visit, as well as use of medications and nutritional supplements. The International Physical Activity Questionnaire (IPAQ) ${ }^{(34,35)}$ and the Harvard-Willett $\mathrm{FFQ}^{(36)}$ were administered at baseline and at 12 months to estimate physical activity status and to determine nutrient intakes, respectively. Participants were asked to report the time they spent on specific types of physical activities in the last $7 \mathrm{~d}$. Metabolic equivalents were calculated based on the activity type as per the IPAQ guidelines ${ }^{(37)}$. A trained research member administered the FFQ, which was based on the participant's usual intake in the last 3 months. Multiple-pass $24-\mathrm{h}$ recalls ${ }^{(38)}$ were administered at each on-site visit and at each monthly telephone follow-up. FFQ data were analysed using the Canadian Nutrient File 2010b to calculate energy, Ca and vitamin D intakes. The 24-h recall data were analysed using Nutritionist Pro software (Axxya Systems) to calculate dietary $\mathrm{Ca}$ and vitamin $\mathrm{D}$ intakes and monitor adherence to the dietary interventions. Supplementation compliance was assessed by reported tablet count during monthly follow-up calls and by verification of reported tablet count upon return of supplement bottles. General health status, use of new medications and nutritional supplements were also surveyed during monthly follow-ups.

Anthropometric measurements. Height, weight and waist and hip circumferences were measured at each visit using standard practices. Standing height was measured to the nearest $0 \cdot 1 \mathrm{~cm}$ using a wall-mounted stadiometer (Seca 242), and weight was measured to the nearest $0 \cdot 1 \mathrm{~kg}$ in light clothing without shoes (Tanita TBF-310; Tanita Corp.). Waist circumference was measured to the nearest $0.5 \mathrm{~cm}$ at the midpoint between the lower costal margin and the iliac crest according to Health Canada guidelines $^{(39)}$. Hip circumference was measured to the nearest $0 \cdot 1 \mathrm{~cm}$ at the level of the symphysis pubis and the greatest gluteal protuberance based on the protocol followed by Health Canada, the Canadian Standard Test of Fitness, 3rd edition ${ }^{(40)}$.

Vascular measurements. Peripheral resting blood pressure was measured using the automated BpTRU device (BpTRU Medical Devices Inc.), according to the Canadian Hypertension Education Program guidelines ${ }^{(41)}$. Arterial stiffness assessments were performed with the participant in the supine position after a 10 -min rest period in a quiet, temperature-controlled $\left(20 \pm 1^{\circ} \mathrm{C}\right)$ and humidity-controlled $(60 \pm 5 \%)$ environment (Vascular Health Unit, McGill University Health Centre, Director: S. S. D. co-author). Applanation tonometry (SphygmoCor, AtCor Medical) was used to measure carotid-femoral pulse wave velocity (cfPWV), the gold standard measurement assessing central arterial stiffness, as well as carotid-radial pulse wave velocity (crPWV), which is a measure of peripheral stiffness. Specifically, cfPWV and crPWV were measured in triplicates (and averaged out) using a high-fidelity micromanometer on the tip of a hand-held tonometer (SPC-301; Millar Instruments), which was applied over the carotid and femoral (or radial) arteries, and a 3-lead electrocardiogram. To minimise variability between replicate measurements, we discarded PWV measures that had consecutive readings with a difference that was $>0.5 \mathrm{~m} / \mathrm{s}^{(42)}$. PWV measures with individual-site (carotid or femoral) electrocardiogram (ECG)-pulse transit time SD $>5 \%$ and overall SD $>10 \%$ were discarded and the measurement was repeated. PWV with a heart rate difference between the carotid and the femoral site of $\geq 5$ beats/min were also rejected, and the measurement was repeated. Only high-quality measurements were accepted. After measuring the distance between the recording sites, the PWV was calculated (PWV=distance $(\mathrm{m}) /$ transit time $(s))^{(43-47)}$. To minimise the effect of the circadian cycle, assessments were performed at the same time in the morning at each visit (online Supplementary Table S1).

Biomarkers. Fasting blood samples were collected between 07.00 and 10.00 hours at baseline and final visits to minimise any variation owing to biological rhythms of most biomarkers. Ionised $\mathrm{Ca}$ was analysed within $30 \mathrm{~min}$ of sampling at the Division of Biochemistry at the Montreal General Hospital (MGH) using the ABL 800 series blood gas analyser (Radiometer America), with an intra-individual \% CV of $2 \cdot 8 \%$ based on internal quality controls for ionised $\mathrm{Ca}$. The remaining blood samples were separated into plasma and serum fractions and stored at $-80^{\circ} \mathrm{C}$ until further analysis. Measurements of total cholesterol, HDL-cholesterol, TAG, apo-A1 and apo-B, and high-sensitivity C-reactive protein (hsCRP) were performed using Synchron LX Systems (Beckman Coulter Inc.) at the end of the trial period in one single batch at the MGH. The intra-assay \% CV was $0.7 \%$ for total cholesterol, $0.6 \%$ for TAG, $1.5 \%$ for HDL-cholesterol, $0.9 \%$ for apo-A1, $1 \%$ for apo-B and $0.9 \%$ for hsCRP. LDL-cholesterol was calculated using the Friedewald equation ${ }^{(48)}$. Plasma total 25-hydroxy vitamin D $(25(\mathrm{OH}) \mathrm{D})$ and parathyroid hormone $(\mathrm{PTH})$ concentrations were measured using an autoanalyzer (Liaison, DiaSorin) with an intra-assay CV of 4.1 and $3.6 \%$, respectively. All 25(OH)D and PTH measurements were performed at the School of Dietetics and Human Nutrition of McGill University, which participated in the international Vitamin D External Quality Assessment Program (http://www.deqas.org) and obtained a certificate of proficiency for 2011-2015 (Director: H. A. W. - co-author). The accuracy of the methodology for vitamin D assays was assessed using the National Institutes of Standards and Technology Standard Reference Material (972a, level 1 and level 2). The accuracy of Liaison controls were $94 \cdot 4-100 \cdot 4 \%$ for $25(\mathrm{OH}) \mathrm{D}$ and $96 \cdot 5-97 \cdot 8 \%$ for PTH.

Online feedback survey. Following completion of this pilot trial, all participants were invited to complete an online feedback survey - 'Participation in Pilot Calcium Study: Feedback Survey' - anonymously using LimeSurvey application (http://www.limesurvey.org). The survey aimed to evaluate the acceptability of the trial interventions, participant satisfaction with the dietary modifications, supplement regimen, trial visits and procedures, as well as monthly contacts with the dietitian. The survey included an open-ended question for the participants' feedback on the strengths and limitations of the trial design. 


\section{Statistical analyses}

Summary statistics were computed for baseline characteristics, presented as mean values and standard deviations for normally distributed continuous variables or as counts and percentages for categorical variables. The $\chi^{2}$ test was used to test for differences in proportions. Independent Student's $t$ tests were used to compare the differences in mean intakes of dietary $\mathrm{Ca}$ and vitamin $\mathrm{D}$ at 1,6 and 12 months between the two intervention groups. Independent Student's $t$ tests were also used to compare the differences in blood pressure, anthropometric measurements, physical activity, vascular and bone biomarkers at 12 months and over time. Within- and between-group differences over time in cfPWV were examined using repeated-measures ANOVA, as per intention-to-treat analysis. Owing to the exploratory nature of the trial and small sample size, no multiple-testing correction was performed $^{(49)}$. Significance was set at $P<0 \cdot 05$. All statistical analyses were performed using the statistical software package SPSS version 22 for Windows (SPSS Inc.).

\section{Results}

The Consolidated Standards of Reporting Trials (CONSORT) flow chart depicting participant selection process is demonstrated in Fig. 1. In total, thirteen women were enrolled for the trial between July 2012 and February 2013 at a mean age of 63.2 (SD 6.6) years, BMI of 24.6 (SD 2.9$) \mathrm{kg} / \mathrm{m}^{2}$, systolic blood pressure of 111.1 (SD 13.0) $\mathrm{mmHg}$ and diastolic blood pressure of 71.3 (sD 10.1) mmHg. Among them, seven participants were randomised to the CaSuppl group and six participants to the CaDiet group. In total, three participants withdrew from the trial

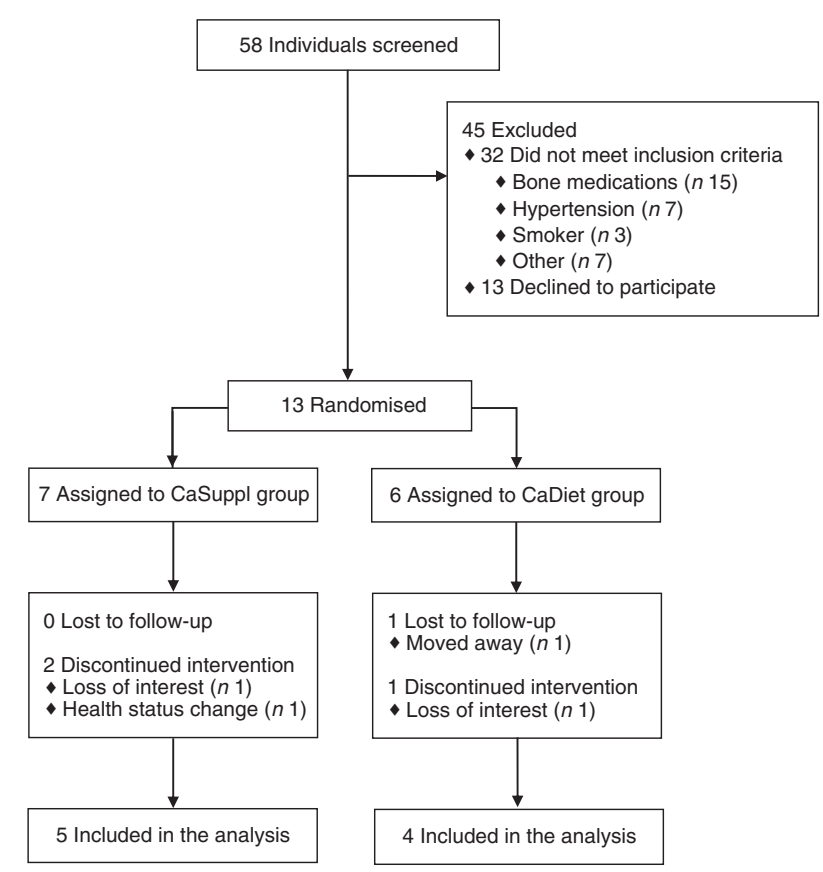

Fig. 1. Consolidated Standards of Reporting Trials (CONSORT) diagram depicting the flow of participants. CaSuppl, calcium supplement group; CaDiet, calcium diet group. and one was lost to follow-up. Dropout rates were similar between groups. Of the four participants who did not complete the trial, one withdrew from the trial when her family doctor initiated her on antihypertensive medication within 2 months following randomisation; her blood pressure at randomisation was within normal limits. We were unable to ascertain her blood pressure as she did not return for on-site visits and we were unsuccessful in contacting her physician.

No differences in baseline characteristics were observed between the two groups with regard to age, anthropometric variables, physical activity level, nutrient intakes including $\mathrm{Ca}$ and vitamin $\mathrm{D}$, or vascular variables (Table 1 ). The only statistically significant difference between groups was a higher reported family history of stroke in the CaSuppl group $(P=0.03)$.

In all, nine participants completed all telephone follow-ups and on-site visits. Dietary data derived from the multiple-pass 24-h recalls indicated that both the CaSuppl group and the CaDiet group participants were within $20 \%$ of their calculated $\mathrm{Ca}$ intake target over the trial period (94-113 and 96-116\%, respectively). The calculated average dietary $\mathrm{Ca}$ intake from the multiple-pass 24-h recalls indicated that the CaSuppl group had significantly lower mean intakes ( 480 (SD 292) mg/d at 1 month, 400 (SD 70) mg/d at 6 months and 600 (sD 93) mg/d at 12 months) than the CaDiet group (1380 (SD 437) $\mathrm{mg} / \mathrm{d}$ at 1 month, 1269 (sD 188) mg/d at 6 months and 1019 (SD 323) $\mathrm{mg} / \mathrm{d}$ at 12 months $)(P<0.05$; Fig. 2). During the 12-month intervention, the CaSuppl group had a significantly lower intake of dietary vitamin D at 6 months $(1.54 v .11 .8 \mu \mathrm{g})(P<0.05)$. The 12-month average total Ca intake (dietary + supplemental $\mathrm{Ca}$ ) in the CaSuppl group was $1124 v .1242 \mathrm{mg} / \mathrm{d}$ in the CaDiet group, and we found no significant differences $(P=0 \cdot 13)$. When we compared the usual dietary $\mathrm{Ca}$ intake data derived from the Harvard-Willett FFQ, there was a significant between-group difference at 12 months $(P=0 \cdot 04)$. However, the mean dietary Ca intake was 761 (sD 277) mg/d in the CaSuppl group and 1187 (sD 232) $\mathrm{mg} / \mathrm{d}$ in the CaDiet group. Mean dietary vitamin D intakes derived from the FFQ method at 12 months were 3.42 (sD 1.50) $\mu \mathrm{g} / \mathrm{d}$ and 10.43 (sD 4.28) $\mu \mathrm{g} / \mathrm{d}$ in the CaSuppl and the CaDiet groups, respectively. Overall adherence rate to $\mathrm{Ca}$ supplements was $85 \%$ in the CaSuppl group, and adherence rate to vitamin D supplements was $98 \%$ in both groups. None of the participants reported adverse events from supplements.

We found no significant differences in body weight, BMI, waist circumference, waist:hip ratio or physical activity level between the intervention groups at 12 months, and no significant within-group change over time was observed $(P>0.05)$.

Following the 12-month intervention, no significant differences in blood pressure or vascular health biomarkers were found between groups or within groups over time (Table 2). We observed that the systolic blood pressure was higher in the CaSuppl group compared with the CaDiet group at 12 months, although not reaching statistical significance $(P=0.05)$. Systolic blood pressure values were normal in both groups at 12 months. Similarly, we observed a mean increase of $8.6 \mathrm{mmHg}$ in diastolic blood pressure in the CaSuppl group $v$. a mean increase of $1 \mathrm{mmHg}$ in the CaDiet group, but the between group change over time was not statistically significant $(P=0 \cdot 07)$. Diastolic blood pressure values were normal in both 
Table 1. Baseline characteristics of thirteen participants by intervention group (Mean values and standard deviations; number of participants and percentages)

\begin{tabular}{|c|c|c|c|c|c|}
\hline \multirow[b]{2}{*}{ Characteristics } & \multicolumn{2}{|c|}{ CaSuppl $(n 7)$} & \multicolumn{2}{|c|}{ CaDiet $(n 6)$} & \multirow[b]{2}{*}{$P$} \\
\hline & Mean & SD & Mean & SD & \\
\hline Age (years) & $65 \cdot 0$ & $4 \cdot 2$ & $60 \cdot 8$ & 8.5 & 0.26 \\
\hline Caucasian & & & & 0.31 \\
\hline$n$ & \multicolumn{2}{|c|}{4} & \multirow{2}{*}{\multicolumn{2}{|c|}{$\begin{array}{c}5 \\
83 \cdot 3\end{array}$}} & \\
\hline$\%$ & \multicolumn{2}{|c|}{$57 \cdot 1$} & & & \\
\hline Menarche age (years) & $13 \cdot 0$ & 1.4 & $12 \cdot 5$ & 1.87 & 0.59 \\
\hline Menopause age (years) & $49 \cdot 3$ & $5 \cdot 8$ & 47.5 & $6 \cdot 8$ & 0.62 \\
\hline BMI $\left(\mathrm{kg} / \mathrm{m}^{2}\right)$ & $24 \cdot 6$ & $2 \cdot 8$ & $24 \cdot 6$ & $3 \cdot 33$ & 0.10 \\
\hline Waist circumference $(\mathrm{cm})$ & $80 \cdot 8$ & $8 \cdot 4$ & $81 \cdot 7$ & $8 \cdot 11$ & 0.85 \\
\hline Hip circumference $(\mathrm{cm})$ & $97 \cdot 8$ & 7.9 & 98.6 & $2 \cdot 4$ & 0.80 \\
\hline Waist:hip ratio & 0.83 & 0.03 & 0.83 & 0.07 & 0.98 \\
\hline \multicolumn{6}{|l|}{ Blood pressure } \\
\hline Systolic (mmHg) & 114 & 16 & 108 & 8 & 0.38 \\
\hline Diastolic (mmHg) & 70 & 13 & 73 & 5 & 0.71 \\
\hline \multicolumn{6}{|l|}{ Arterial stiffness } \\
\hline $\operatorname{cfPWV}(\mathrm{m} / \mathrm{s})$ & 7.68 & 1.4 & $7 \cdot 87$ & $2 \cdot 2$ & 0.85 \\
\hline crPWV (m/s) & 7.67 & 0.8 & $8 \cdot 16$ & 1.4 & 0.43 \\
\hline iCalcium (mmol/l) & 1.26 & 0.3 & 1.27 & 0.3 & 0.56 \\
\hline 25(OH)D (nmol/l) & $60 \cdot 2$ & $25 \cdot 2$ & 63.4 & $36 \cdot 0$ & 0.85 \\
\hline PTH (pmol/l) & $31 \cdot 1$ & $11 \cdot 1$ & $24 \cdot 7$ & $11 \cdot 2$ & 0.33 \\
\hline \multicolumn{6}{|l|}{ Family history } \\
\hline Osteoporosis & \multirow{2}{*}{\multicolumn{2}{|c|}{4}} & \multirow{2}{*}{\multicolumn{2}{|c|}{2}} & 0.39 \\
\hline$n$ & & & & & \\
\hline$\%$ & \multicolumn{2}{|c|}{$57 \cdot 1$} & \multicolumn{2}{|c|}{$33 \cdot 3$} & \\
\hline $\mathrm{CHD}$ & & & 0.39 \\
\hline$n$ & \multirow{2}{*}{\multicolumn{2}{|c|}{$\begin{array}{c}4 \\
57 \cdot 1\end{array}$}} & \multicolumn{2}{|c|}{$\begin{array}{c}2 \\
33 \cdot 3\end{array}$} & \\
\hline$\%$ & & & & \\
\hline Stroke & & & & 0.03 \\
\hline$n$ & \multicolumn{2}{|c|}{4} & \multicolumn{2}{|c|}{0} & \\
\hline$\%$ & \multicolumn{2}{|c|}{$57 \cdot 1$} & & & \\
\hline Dyslipidaemia & & & & & 0.14 \\
\hline$n$ & & & & & \\
\hline$\%$ & & & & & \\
\hline Hypertension & & & & & 0.14 \\
\hline$n$ & & & & & \\
\hline$\%$ & & & & & \\
\hline Diabetes & & & & & 0.73 \\
\hline$n$ & & & & & \\
\hline$\%$ & & & & & \\
\hline Physical activity (MET-min/week) & 3320 & 1502 & 6044 & 6150 & 0.33 \\
\hline Dietary intake $(\text { per } d)^{\star}$ & & & & & \\
\hline Energy (kJ) & 10496 & 2717 & 9471 & 2378 & 0.49 \\
\hline $\mathrm{Ca}(\mathrm{mg})$ & 1054 & 317 & 1170 & 127 & 0.42 \\
\hline Vitamin D $(\mu \mathrm{g})$ & $15 \cdot 6$ & $10 \cdot 2$ & $12 \cdot 3$ & $2 \cdot 22$ & 0.47 \\
\hline
\end{tabular}

CaSuppl, Ca supplement group; CaDiet, Ca diet group; cfPWV, carotid-femoral pulse wave velocity; crPWV, carotid-radial pulse wave velocity; iCalcium, ionised Ca; 25(OH)D, 25-hydroxyvitamin D; PTH, parathyroid hormone; MET, metabolic equivalent.

* Dietary data derived from the Harvard-Willett semi-quantitative FFQ.

(a)

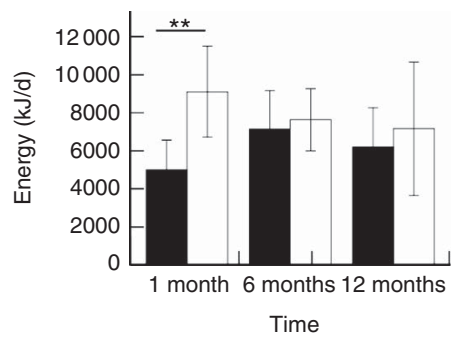

(b)

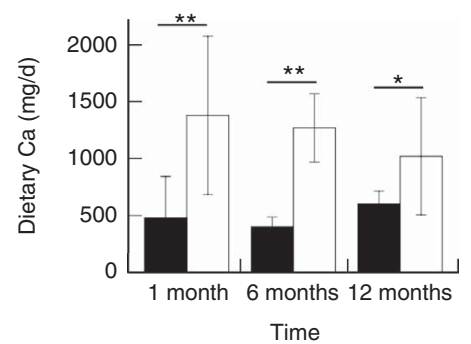

(c)

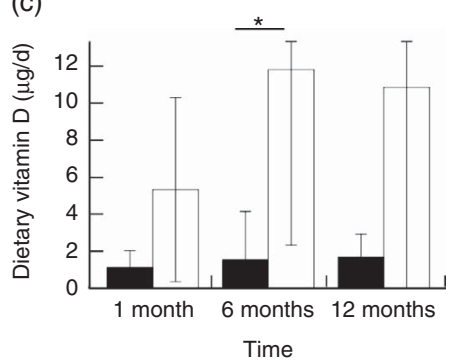

Fig. 2. Mean energy (a), dietary calcium (b) and dietary vitamin $\mathrm{D}$ (c) intake over time. Values are means, with $95 \% \mathrm{Cl}$ represented by vertical bars. Mean value was significantly different from that of the calcium diet group (CaDiet): ${ }^{\star} P<0.05,{ }^{\star \star} P<0.01$. $\square$, Ca supplement group; $\square$, CaDiet. 
groups at 12 months. Arterial stiffness measurements were completed with a mean duration of $<45 \mathrm{~min}$ with each participant at each visit without adverse reactions. No betweengroup differences in cfPWV or crPWV were observed over time (Fig. 3). A significant increase in crPWV was observed between 6 and 12 months in the CaDiet group $(P=0.03)$.

Plasma lipid concentrations did not differ significantly between CaSuppl and CaDiet following the 12-month intervention (Table 2). No significant difference in change over time between groups was observed with the exception of HDL-cholesterol, for which there was an increase of $0.20 \mathrm{mmol} / \mathrm{l}$ in CaSuppl and a decrease of $0.15 \mathrm{mmol} / 1$ in CaDiet $(P=0.02)$. Levels of apo-A1, apo-B and hsCRP did not differ significantly between intervention groups. No between-group differences at 12 months were observed in markers of bone health including ionised $\mathrm{Ca}, 25(\mathrm{OH}) \mathrm{D}, \mathrm{PTH}$ and phosphate. There was a decrease in ionised $\mathrm{Ca}$ of $0.03 \mathrm{mmol} / \mathrm{l}(P=0.007)$ and an increase of $0.57 \mathrm{pmol} / \mathrm{l}$ in PTH $(P<0.001)$ between baseline and end of trial in the CaDiet group. A mean decrease of $3.70 \mathrm{nmol} / 1$ in $25(\mathrm{OH}) \mathrm{D}$ level was also observed in the CaDiet group, but this was not statistically significant $(P=0.33)$ (Table 3).
Responses from the online survey ( $n$ 8) showed that all participants found the adherence to daily supplementation easy. Although only $25 \%$ of the CaDiet group reported having difficulty meeting the daily dietary $\mathrm{Ca}$ target of three portions of dairy products and alternatives, all the participants from the CaSuppl group found it challenging to adhere to the dietary restriction of one small portion of Ca-rich food a day. Reasons provided by CaSuppl participants encountering difficulties included 'not being able to eat some favourite foods' or had to 'reduce some of the regularly consumed foods significantly'. Moreover, one participant felt that 'it was difficult to measure some foods' to adequately report the portion consumed during telephone follow-ups. Overall, all participants felt adequately informed on how to modify their intake of dietary $\mathrm{Ca}$ from the baseline nutritional education session and the monthly follow-ups.

\section{Discussion}

Our pilot trial demonstrated the feasibility of conducting a large-scale, randomised-controlled trial to estimate the effect of supplemental $\mathrm{Ca}$ as compared with dietary $\mathrm{Ca}$ on vascular and bone health in postmenopausal women. In particular, we

Table 2. Changes in blood pressure and vascular health biomarkers from baseline to the end of the trial (12 months)

(Mean values and standard deviations)

\begin{tabular}{|c|c|c|c|c|c|c|c|c|c|c|}
\hline & \multicolumn{4}{|c|}{ CaSuppl ( $n$ 5) } & \multicolumn{4}{|c|}{ CaDiet $(n 4)$} & \multirow{3}{*}{$\begin{array}{c}P \text { (CaSuppl } v . \\
\text { CaDiet at } 12 \text { months) }\end{array}$} & \multirow{3}{*}{$\begin{array}{c}P(\Delta \text { CaSuppl } \\
\Delta \text { CaDiet })\end{array}$} \\
\hline & \multicolumn{2}{|c|}{ Baseline } & \multicolumn{2}{|c|}{12 months } & \multicolumn{2}{|c|}{ Baseline } & \multicolumn{2}{|c|}{12 months } & & \\
\hline & Mean & SD & Mean & SD & Mean & SD & Mean & SD & & \\
\hline $\operatorname{cfPWV}(\mathrm{m} / \mathrm{s})$ & $8 \cdot 25$ & 1.09 & $8 \cdot 70$ & 1.80 & $8 \cdot 71$ & $2 \cdot 08$ & $7 \cdot 30$ & $1 \cdot 10$ & 0.24 & 0.16 \\
\hline $\operatorname{crPWV}(\mathrm{m} / \mathrm{s})$ & 7.45 & 0.82 & 7.60 & 0.90 & 8.55 & 1.45 & 8.60 & 0.90 & 0.13 & 0.93 \\
\hline $\mathrm{SBP}(\mathrm{mmHg})$ & $113 \cdot 6$ & $18 \cdot 8$ & $125 \cdot 0$ & $15 \cdot 0$ & 103.8 & $6 \cdot 8$ & 104.0 & $9 \cdot 0$ & 0.05 & $0 \cdot 10$ \\
\hline DPB (mmHg) & 67.4 & $14 \cdot 1$ & $76 \cdot 0$ & 14.0 & $70 \cdot 8$ & $5 \cdot 1$ & $70 \cdot 0$ & $6 \cdot 0$ & 0.46 & 0.07 \\
\hline Cholesterol (mmol/l) & $5 \cdot 39$ & 1.02 & $5 \cdot 78$ & 0.51 & $6 \cdot 14$ & 0.38 & $6 \cdot 10$ & 0.84 & 0.50 & 0.38 \\
\hline TAG $(\mathrm{mmol} / \mathrm{l})$ & 0.93 & 0.56 & 1.22 & 0.87 & 0.88 & 0.28 & 0.80 & 0.38 & 0.40 & 0.20 \\
\hline HDL-cholesterol $(\mathrm{mmol} / \mathrm{l})$ & 1.78 & 0.77 & 1.98 & 0.81 & 1.99 & 0.53 & 1.84 & 0.41 & 0.76 & 0.02 \\
\hline LDL-cholesterol $(\mathrm{mmol} / \mathrm{l})$ & $3 \cdot 19$ & 0.98 & 3.25 & 0.61 & 3.00 & 1.95 & 3.90 & 1.04 & 0.28 & 0.27 \\
\hline Apo-A1 (g/l) & 1.31 & 0.75 & 1.76 & 0.33 & 1.65 & 0.35 & 1.59 & 0.28 & 0.45 & 0.11 \\
\hline Apo-B $(\mathrm{g} / \mathrm{l})$ & 1.04 & 0.33 & 1.07 & 0.26 & $1 \cdot 17$ & 0.24 & 1.20 & 0.32 & 0.55 & 1.00 \\
\hline hsCRP (mg/l) & 1.68 & 1.71 & 0.76 & 0.49 & 0.33 & 0.15 & 0.38 & 0.24 & $0 \cdot 19$ & 0.21 \\
\hline
\end{tabular}

cfPWV, carotid-femoral pulse wave velocity; crPWV, carotid-radial pulse wave velocity; SBP, systolic blood pressure; DBP, diastolic blood pressure; hsCRP, high-sentivity C-reactive protein; $\Delta$, before and after intervention comparisons.

(a)

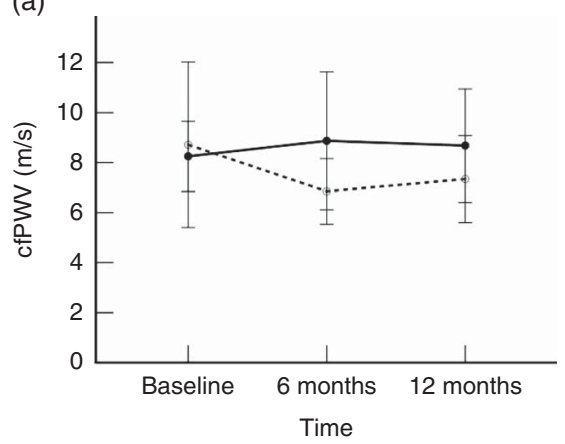

(b)

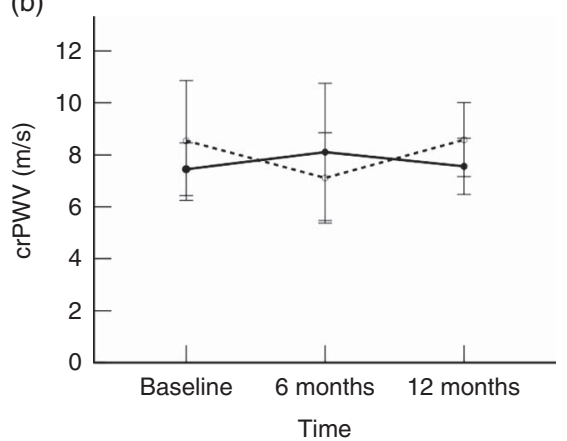

Fig. 3. Change in arterial stiffness markers, (a) carotid-femoral pulse wave velocity (cfPWV) $\left(P_{\text {time }} \times\right.$ intervention $\left.=0 \cdot 16\right)$ and $(\mathrm{b})$ carotid-radial pulse wave velocity (crPWV) $\left(P_{\text {time } \times \text { intervention }}=0.93\right)$, over time. Values are means, with $95 \% \mathrm{Cl}$. $\longrightarrow$, Calcium supplement group;,---- calcium diet group. 
Table 3. Changes in ionised calcium (iCalcium), 25-hydroxyvitamin $\mathrm{D}(25(\mathrm{OH}) \mathrm{D})$ and parathyroid hormone (PTH) from baseline to the end of the trial (12 months) (Mean values and standard deviations)

\begin{tabular}{|c|c|c|c|c|c|c|c|c|c|c|}
\hline & \multicolumn{4}{|c|}{ CaSuppl (n 5) } & \multicolumn{4}{|c|}{ CaDiet $(n 4)$} & \multirow{3}{*}{$\begin{array}{c}P \text { (CaSuppl } v . \\
\text { CaDiet at } 12 \text { months) }\end{array}$} & \multirow{3}{*}{ 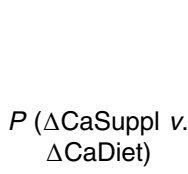 } \\
\hline & \multicolumn{2}{|c|}{ Baseline } & \multicolumn{2}{|c|}{12 months } & \multicolumn{2}{|c|}{ Baseline } & \multicolumn{2}{|c|}{12 months } & & \\
\hline & Mean & SD & Mean & SD & Mean & SD & Mean & SD & & \\
\hline iCalcium (mmol/l) & 1.25 & 0.04 & 1.26 & 0.03 & $1 \cdot 28$ & 0.03 & $1 \cdot 25$ & 0.03 & 0.65 & 0.06 \\
\hline $25(\mathrm{OH}) \mathrm{D}(\mathrm{nmol} / \mathrm{l})$ & $54 \cdot 1$ & $26 \cdot 6$ & 63.6 & 13.9 & $82 \cdot 8$ & $22 \cdot 0$ & 79.1 & $25 \cdot 4$ & 0.28 & 0.23 \\
\hline PTH (pmol/l) & 3.50 & 1.33 & 2.89 & 0.72 & $2 \cdot 27$ & 0.29 & 2.84 & 0.29 & 0.93 & 0.06 \\
\hline Phosphate $(\mathrm{mmol} / \mathrm{l})$ & 1.30 & 0.16 & 1.27 & 0.11 & 1.23 & 0.26 & 1.29 & 0.17 & 0.86 & 0.49 \\
\hline
\end{tabular}

$\Delta$, Before and after intervention comparisons

demonstrated that the combination of an initial in-person nutritional counselling session and monthly telephone follow-ups was effective to ensure participant adherence to the dietary aspects of the trial protocol. Participants felt that the monthly contacts with the dietitian were efficient to help them maintain the proper dietary $\mathrm{Ca}$ intake throughout the trial. In addition, we were able to demonstrate the feasibility in conducting the visits and a comprehensive set of vascular tests without adverse reactions or unforeseen problems.

Preliminary results from this pilot trial did not show any differential effects of $\mathrm{Ca}$ supplementation as compared with dietary $\mathrm{Ca}$ on arterial stiffness or on vascular health biomarkers. There was a significant increase in crPWV from 6 to 12 months in the CaDiet group. These observations may suggest an increase in peripheral stiffness, but have no clinical significance on aortic stiffness, which is the gold standard of arterial stiffness ${ }^{(50)}$. It is noteworthy that the values of vascular health markers (cfPWV and blood pressure) were well within normal limits in our study group, at all time points ${ }^{(51,52)}$. However, the results have to be interpreted with caution given the small sample size.

The rigorous design of our trial differentiates it from other randomised-controlled trials that investigated the effect of $\mathrm{Ca}$ supplements on vascular and bone health. None of the previous trials controlled for dietary intake of Ca despite the reported administration of $1000-1200 \mathrm{mg} / \mathrm{d}$ of elemental Ca to treatment groups $^{(6,16,53-55)}$. In our trial, a dietitian evaluated baseline Ca and vitamin D intakes and provided early nutritional education to each participant at the initiation of the trial to ensure a total daily intake of $1200 \mathrm{mg}$ Ca either from dietary sources alone or predominantly from $\mathrm{Ca}$ supplements. The regular monthly follow-ups allowed close monitoring of protocol adherence and further counselling to ensure adherence to the trial protocol. We observed a high compliance of $85 \%$ to Ca supplements, whereas comparable trials reported compliance rates between 59 and $85 \%^{(6,16,53-55)}$. We also observed that the rigorous design of our pilot trial was effective to help achieve the target dietary $\mathrm{Ca}$ in each group at monthly follow-ups. However, the CaSuppl average dietary Ca intake estimated from the Harvard-Willett FFQ at 12 months was above the assigned Ca target (761 v. $450 \mathrm{mg} / \mathrm{d})$. This observed difference may be a result of over-reporting by one participant during the final visit. Given the small sample size, the mean value is expected to be influenced by one single result. Furthermore, a Ca-focused FFQ may likely be less challenging conceptually for the participant, reduce the respondent burden and increase the accuracy of dietary $\mathrm{Ca}$ intake. Milk and dairy products were most often consumed by the participants in this feasibility trial to meet their intervention targets. Although the Harvard-Willett FFQ includes a section to assess the intake of milk and dairy products, it does not discriminate soft or semi-soft cheeses from firm or hard cheeses. Similarly, this semi-quantitative FFQ does not include Ca-rich items that are currently commonly consumed, such as Greek yogurts, beverages enriched with $\mathrm{Ca}$, canned salmon with bones, pizza and mixed dishes prepared with milk or dairy products. Given the popular consumption of these listed items by the participants in our feasibility trial, a short comprehensive Ca-focused semi-quantitative FFQ may improve the estimation of usual dietary $\mathrm{Ca}$ intake, and likely decrease respondent burden.

Thus far, observational and ancillary studies have investigated the possible relationship between Ca supplements and cardiovascular events following the publication of a meta-analysis of $\mathrm{Ca}$ intervention trials ${ }^{(13)}$ and a secondary analysis of the Women's Health Initiative $\mathrm{Ca} /$ vitamin $\mathrm{D}$ supplementation study's data set ${ }^{(56)}$. More specifically, Bolland et al. ${ }^{(13)}$ demonstrated an increased risk of myocardial infarction in individuals who received Ca supplements alone (hazard ratio (HR) 1.31 ; $95 \%$ CI 1.02, 1.67) or with vitamin $\mathrm{D}^{(56)}$ (HR 1.21; $95 \%$ CI 1.01, 1.44) based on self-reported and verified myocardial infarctions. In contrast, a recent meta-analysis of published and unpublished results from randomised-controlled trials of women only by Lewis et al. ${ }^{(57)}$ demonstrated an absence of increased risk for CHD (relative risk (RR) 1.02; $95 \%$ CI $0.96,1.09$ ) or all-cause mortality (RR 0.96; $95 \%$ CI 0.91, 1.02) from Ca supplements with or without vitamin $D$ in elderly women. Yet, the evidence remains conflicting, which may be due to the primary investigated end point of randomised-controlled trials not being related to cardiovascular risks and an oversight on the assessment of dietary $\mathrm{Ca}$ intakes $^{(17,18,57-59)}$.

Observational studies suggest an association between higher serum Ca concentrations and carotid artery plaque thickness ${ }^{(60)}$, increased risk of myocardial infarction and stroke ${ }^{(61)}$ in older men and women. An increase in total serum Ca level of $0 \cdot 1 \mathrm{mmol} / \mathrm{l}$ has been reported to be associated with $23 \%$ higher odds of abdominal aortic calcification in postmenopausal women $^{(62)}$. Furthermore, it has been previously shown that 
ionised $\mathrm{Ca}$ concentrations increase acutely following supplementation with $1000 \mathrm{mg}$ of $\mathrm{Ca}^{(63,64)}$, although whether these observed changes are maintained with chronic $\mathrm{Ca}$ supplement use is unknown. Reid et al. speculate that the increase in serum ionised $\mathrm{Ca}$ concentrations may lead to a sequence of events contributing to the acceleration of vascular disease by $\mathrm{Ca}$ supplementation. They hypothesised that the pathogenic pathway leading to progressive arterial calcification is via a loss of inhibition of mineralisation due to increased complexing of ionised $\mathrm{Ca}$ with pyrophosphate ${ }^{(65)}$, reduced inhibition of arteriosclerotic signalling in vascular smooth muscle cells due to decreased PTH levels ${ }^{(58)}$ and increased binding to Ca-sensing receptors on vascular smooth muscle cells and platelets ${ }^{(65)}$. Increased arterial stiffness and impaired endothelial vasodilator function resulting from high circulating levels of ionised $\mathrm{Ca}$ associated with $\mathrm{Ca}$ supplementation could also lead to vascular damage ${ }^{(58)}$. However, Burt et al. ${ }^{(64)}$ examined the acute effect of $1000 \mathrm{mg}$ of $\mathrm{Ca}$ citrate on arterial stiffness in adults aged 50 years and above and found no significant change in PWV $3 \mathrm{~h}$ following supplementation.

Arterial stiffness, as measured by the 'gold-standard' cfPWV, is an overall indicator of vascular health and is strongly associated with the development of atherosclerosis at different sites of the arterial system and CVD and cardiovascular events $^{(28,29,66,67)}$. An increase in cfPWV by $1 \mathrm{~m} / \mathrm{s}$ corresponds to an adjusted risk increase of 14,15 and $15 \%$ in total cardiovascular events, cardiovascular mortality and all-cause mortality, respectively ${ }^{(66)}$. Using this gold standard measure, dairy food intake has been shown to have a favourable effect on arterial stiffness, as well as overall cardiovascular profile and reduced mortality ${ }^{(68-71)}$. However, the evidence regarding the effect of $\mathrm{Ca}$ in the form of supplements on arterial stiffness is less consistent. When administered to healthy volunteers, Ca citrate (single oral dose of $1000 \mathrm{mg}$, measurements at times 0 , 60,120 and $180 \mathrm{~min}$ ) was observed to cause an acute increase in total and ionised $\mathrm{Ca}$ (by an average of 0.10 and $0.06 \mathrm{mmol} / \mathrm{l}$, respectively) and a decrease in $\mathrm{PTH}^{(64)}$. However, the acute increase in $\mathrm{Ca}$ was associated with reduced arterial wave reflection, which in the long-term may reduce cardiovascular risk $^{(66)}$. In an acute loading cross-over trial, $600 \mathrm{mg}$ of Ca citrate and $600 \mathrm{mg}$ of $\mathrm{Ca}$ from dairy products did not produce any differential effect on arterial stiffness in young, healthy subjects $2 \mathrm{~h}$ after each challenge ${ }^{(72)}$. In our pilot trial, Ca supplements did not affect blood pressure, cfPWV or crPWV differently than dietary Ca throughout the 12-month intervention.

The effect of $\mathrm{Ca}$ supplementation on serum lipids is inconsistent in the literature. Some studies report that supplemental Ca may cause beneficial changes in circulating lipids ${ }^{(73,74)}$, whereas several studies found no effect $^{(75-78)}$. In contrast, Ca supplementation as compared with placebo for 12 months improved the lipid profile in a randomisedcontrolled trial of postmenopausal women, and this improvement was still observed at the 3-year follow-up ${ }^{(73)}$. Dairy foods have been reported to have either neutral or beneficial effects on blood lipid profile ${ }^{(79-82)}$. In contrast, supplemental $\mathrm{Ca}$ in the form of calcium carbonate did not exert such an effect on blood lipid concentrations ${ }^{(78)}$. In this study, we observed an increase in HDL-cholesterol in the supplement intervention group but no other change in blood lipid profile. An increase in HDL-cholesterol concentration following $\mathrm{Ca}$ supplementation has been reported in the literature ${ }^{(73,83,84)}$, and may likely be a result of the complexing of fatty and bile acids by Ca the intestinal tract ${ }^{(73,85)}$.

Although our trial provides enough information to ascertain the feasibility of a larger randomised-controlled trial, it is limited by its small sample size, and therefore prevents conclusions regarding effects of the $\mathrm{Ca}$ interventions on vascular parameters. Although we experienced a dropout rate of $30 \%$ (four out of thirteen participants), in keeping with other randomised-controlled trials of $\mathrm{Ca}$ interventions ${ }^{(53,73,86)}$, there were no differential dropout rates between the trial groups. Motivational interviewing techniques and participant satisfaction should be emphasised immediately following intervention initiation to improve participant retention, as this pilot trial shows that the first few months were critical times for dropout. An adequately powered clinical trial is warranted to effectively elucidate the effect of supplemental $\mathrm{Ca}$ on vascular health in postmenopausal women, and is currently underway by our group (ClinicalTrials.gov number, NCT01731340). Given that we have modified the procedures based on the online feedback survey of participants after the completion of the pilot trial, we anticipate a lower dropout rate in our ongoing randomised-controlled trial.

In conclusion, the findings of this 12-month pilot trial indicate that both diet modifications and supplemental interventions were associated with high compliance and tolerance, demonstrating the feasibility of the interventions. Although limited by a small sample size, the results suggest that following this 12-month intervention, supplemental Ca does not exert a different effect than dietary $\mathrm{Ca}$ on vascular or bone health markers in healthy postmenopausal women. Nevertheless, the current state of uncertainty warrants further research to assess the effect of supplemental $v$. dietary Ca on the development of CVD disease and whether this effect is mediated through established cardiovascular markers. In this context, results of an adequately powered randomised-controlled trial will facilitate the development of public health recommendations regarding Ca supplementation. Building on our findings of the feasibility of trial interventions, a randomised-controlled trial is currently ongoing by our group.

\section{Acknowledgements}

The authors thank the participants of the trial as well as Euro-Pharma who graciously provided the Ca and vitamin D supplements for this pilot trial.

The present trial was supported by the Fonds de recherche du Québec - Santé (FRQS) - Subvention d'établissement de jeunes chercheurs - 2011. The FRQS had no role in the design, analysis or writing of this article.

All named authors have made substantial contribution to the published manuscript as follows: S. N. M., D. G., S. S. D. and H. A. W. contributed to the design of the trial. M. W. and R. H. contributed to the recruitment of participants, collection of the data and statistical analysis. J. G. also contributed to 
collection of the data and laboratory work. A. M. O. contributed to collection of the data, statistical analysis, interpretation of the data and writing of the manuscript. H. A. W., S. N. M. and S. S. D. also contributed to interpretation of the data and writing of the manuscript. All the authors read and approved the final version of the manuscript.

The authors report no conflicts of interest.

\section{Supplementary material}

For supplementary material/s referred to in this article, please visit http://dx.doi.org/doi:10.1017/S0007114516001677

\section{References}

1. Chung M, Lee J, Terasawa T, et al. (2011) Vitamin D with or without calcium supplementation for prevention of cancer and fractures: an updated meta-analysis for the U.S. Preventive Services Task Force. Ann Intern Med 155, 827-838.

2. Bischoff-Ferrari HA, Willett WC, Wong JB, et al. (2005) Fracture prevention with vitamin D supplementation: a meta-analysis of randomized controlled trials. JAMA 293, 2257-2264.

3. Boonen S, Lips P, Bouillon R, et al. (2007) Need for additional calcium to reduce the risk of hip fracture with vitamin $D$ supplementation: evidence from a comparative metaanalysis of randomized controlled trials. J Clin Endocrinol Metab 92, $1415-1423$.

4. Tang BM, Eslick GD, Nowson C, et al. (2007) Use of calcium or calcium in combination with vitamin $\mathrm{D}$ supplementation to prevent fractures and bone loss in people aged 50 years and older: a meta-analysis. Lancet 370, 657-666.

5. Zhu K \& Prince RL (2012) Calcium and bone. Clin Biochem 45, 936-942.

6. Jackson RD, LaCroix AZ, Gass M, et al. (2006) Calcium plus vitamin D supplementation and the risk of fractures. $N$ Engl J Med 354, 669-683.

7. Institute of Medicine (2011) Dietary Reference Intakes for Calcium and Vitamin D. Washington, DC: National Academy of Sciences.

8. Bailey RL, Dodd KW, Goldman JA, et al. (2010) Estimation of total usual calcium and vitamin D intakes in the United States. J Nutr 140, 817-822.

9. Garriguet D (2011) Bone health: osteoporosis, calcium and vitamin D. Health Rep 22, 7-14.

10. Papaioannou A, Morin S, Cheung AM, et al. (2010) 2010 clinical practice guidelines for the diagnosis and management of osteoporosis in Canada: summary. CMAJ 182, 1864-1873.

11. Foote JA, Murphy SP, Wilkens LR, et al. (2003) Factors associated with dietary supplement use among healthy adults of five ethnicities: the multiethnic cohort study. Am J Epidemiol 157, 888-897.

12. Wallace RB, Wactawski-Wende J, O'Sullivan MJ, et al. (2011) Urinary tract stone occurrence in the Women's Health Initiative (WHI) randomized clinical trial of calcium and vitamin D supplements. Am J Clin Nutr 94, 270-277.

13. Bolland MJ, Avenell A, Baron JA, et al. (2010) Effect of calcium supplements on risk of myocardial infarction and cardiovascular events: meta-analysis. BMJ 341, c3691.

14. Bolland MJ, Grey A, Gamble GD, et al. (2011) Calcium supplements with or without vitamin D and risk of cardiovascular events: reanalysis of the Women's Health Initiative limited access dataset and meta-analysis. BMJ 342, d2040.
15. Shah SM, Carey IM, Harris T, et al. (2010) Calcium supplementation, cardiovascular disease and mortality in older women. Pharmacoepidemiol Drug Saf 19, 59-64.

16. Lewis JR, Calver J, Zhu K, et al. (2011) Calcium supplementation and the risks of atherosclerotic vascular disease in older women: results of a 5-year RCT and a 4.5-year follow-up. J Bone Miner Res 26, 35- 41 .

17. Prentice RL, Pettinger MB, Jackson RD, et al. (2013) Health risks and benefits from calcium and vitamin D supplementation: Women's Health Initiative clinical trial and cohort study. Osteoporos Int 24, 567-580.

18. Lewis JR, Zhu K, Thompson PL, et al. (2014) The effects of 3 years of calcium supplementation on common carotid artery intimal medial thickness and carotid atherosclerosis in older women: an ancillary study of the CAIFOS randomized controlled trial. J Bone Miner Res 29, 534-541.

19. Praagman J, Franco OH, Ikram MA, et al. (2015) Dairy products and the risk of stroke and coronary heart disease: the Rotterdam Study. Eur J Nutr 54, 981-990.

20. Huth PJ \& Park KM (2012) Influence of dairy product and milk fat consumption on cardiovascular disease risk: a review of the evidence. Adv Nutr 3, 266-285.

21. Larsson SC, Virtamo J \& Wolk A (2012) Dairy consumption and risk of stroke in Swedish women and men. Stroke $\mathbf{4 3}$, $1775-1780$

22. Tavani A, Gallus S, Negri E, et al. (2002) Milk, dairy products, and coronary heart disease. J Epidemiol Community Health 56, 471-472.

23. Panagiotakos DB, Pitsavos CH, Zampelas AD, et al. (2010) Dairy products consumption is associated with decreased levels of inflammatory markers related to cardiovascular disease in apparently healthy adults: the ATTICA study. J Am Coll Nutr 29, 357-364.

24. Elwood PC, Givens DI, Beswick AD, et al. (2008) The survival advantage of milk and dairy consumption: an overview of evidence from cohort studies of vascular diseases, diabetes and cancer. J Am Coll Nutr 27, 723s-734s.

25. Michaelsson K, Wolk A, Langenskiold S, et al. (2014) Milk intake and risk of mortality and fractures in women and men: cohort studies. BMJ 349, g6015.

26. World Health Organization (2015) Cardiovascular diseases. http://www.who.int/mediacentre/factsheets/fs317/en/ (accessed July 2015).

27. Stramba-Badiale M, Fox KM, Priori SG, et al. (2006) Cardiovascular diseases in women: a statement from the policy conference of the European Society of Cardiology. Eur Heart J 27, 994-1005.

28. Mancia G, De Backer G, Dominiczak A, et al. (2007) 2007 Guidelines for the management of arterial hypertension: the task force for the management of arterial hypertension of the European Society of Hypertension (ESH) and of the European Society of Cardiology (ESC). Eur Heart J 28, 1462-1536.

29. Laurent S, Boutouyrie P, Asmar R, et al. (2001) Aortic stiffness is an independent predictor of all-cause and cardiovascular mortality in hypertensive patients. Hypertension 37, 1236-1241.

30. Kanis JA, on behalf of the World Health Organization Scientific Group (2008) Assessment of osteoporosis at the primary healthcare level. Technical Report. http://www.shef. ac.uk/FRAX (accessed October 2013).

31. Bauer DC (2014) Calcium supplements and fracture prevention. $N$ Engl J Med 370, 387-388.

32. BC Dairy Foundation (2007) Calcium calculator. http:// bcdairyfoundation.ca/interactive/calcium-calculator (accessed October 2013).

33. Health Canada Bureau of Nutritional Sciences (2011) Using the Nutrition Facts Table - \% Daily Value. Ottawa: Health Canada. 
34. Booth M (2000) Assessment of physical activity: an international perspective. Res Q Exerc Sport 71, S114-S120.

35. Craig CL, Marshall AL, Sjostrom M, et al. (2003) International physical activity questionnaire: 12-country reliability and validity. Med Sci Sports Exerc 35, 1381-1395.

36. Willett WC, Sampson L, Stampfer MJ, et al. (1985) Reproducibility and validity of a semiquantitative food frequency questionnaire. Am J Epidemiol 122, 51-65.

37. International Physical Activity Questionnaire (2005) Guidelines for the data processing and analysis of the International Physical Activity Questionnaire - short and long forms. http:// www.ipaq.ki.se (accessed July 2015).

38. Conway JM, Ingwersen LA, Vinyard BT, et al. (2003) Effectiveness of the US Department of Agriculture 5-step multiple-pass method in assessing food intake in obese and nonobese women. $A m$ J Clin Nutr 77, 1171-1178

39. Health Canada (2003) Canadian guidelines for body weight classification in adults. http://www.hc-sc.gc.ca/fn-an/alt_ formats/hpfb-dgpsa/pdf/nutrition/cg_quick_ref-ldc_rapide_ ref-eng.pdf (accessed October 2013).

40. Fitness Canada (1986) Canadian Standard Test of Fitness (CSTF): Operations Manual, 3rd ed. Ottawa: Fitness and Amateur Sport, Government of Canada

41. Daskalopoulou SS, Khan NA, Quinn RR, et al. (2012) The 2012 Canadian Hypertension Education Program recommendations for the management of hypertension: blood pressure measurement, diagnosis, assessment of risk, and therapy. Can J Cardiol 28, 270-287.

42. AtCor Medical (2006) Software Operator's Guide: Pulse Wave Analysis System SCOR-PX. Sydney: AtCor Medical.

43. Van Bortel LM, Duprez D, Starmans-Kool MJ, et al. (2002) Clinical applications of arterial stiffness, Task Force III: recommendations for user procedures. Am J Hypertens 15, 445-452.

44. Gorgui J, Doonan RJ, Gomez YH, et al. (2013) Carotid endarterectomy improves peripheral but not central arterial stiffness. Eur J Vasc Endovasc Surg 45, 548-553.

45. Doonan RJ, Mutter A, Egiziano G, et al. (2013) Differences in arterial stiffness at rest and after acute exercise between young men and women. Hypertens Res 36, 226-231.

46. Yu A, Giannone T, Scheffler P, et al. (2014) The effect of oral contraceptive pills and the natural menstrual cycle on arterial stiffness and hemodynamics (CYCLIC). J Hypertens 32, 100-107.

47. Doonan RJ, Scheffler P, Yu A, et al. (2011) Altered arterial stiffness and subendocardial viability ratio in young healthy light smokers after acute exercise. PLOS ONE 6, e26151.

48. Friedewald WT, Levy RI \& Fredrickson DS (1972) Estimation of the concentration of low-density lipoprotein cholesterol in plasma, without use of the preparative ultracentrifuge. Clin Chem 18, 499-502.

49. Wason JM, Stecher L \& Mander AP (2014) Correcting for multiple-testing in multi-arm trials: is it necessary and is it done? Trials 15, 364 .

50. Salvi P (2012) Pulse Waves: How Vascular Hemodynamics Affects Blood Pressure. Milan: Springer.

51. Reference Values for Arterial Stiffness' Collaboration (2010) Determinants of pulse wave velocity in healthy people and in the presence of cardiovascular risk factors: 'establishing normal and reference values'. Eur Heart J 31, 2338-2350.

52. Daskalopoulou SS, Rabi DM, Zarnke KB, et al. (2015) The 2015 Canadian Hypertension Education Program recommendations for blood pressure measurement, diagnosis, assessment of risk, prevention, and treatment of hypertension. Can J Cardiol 31, 549-568.

53. Grant AM, Avenell A, Campbell MK, et al. (2005) Oral vitamin $\mathrm{D}_{3}$ and calcium for secondary prevention of low-trauma fractures in elderly people (Randomised Evaluation of
Calcium Or vitamin D, RECORD): a randomised placebocontrolled trial. Lancet 365, 1621-1628.

54. Prince RL, Devine A, Dhaliwal SS, et al. (2006) Effects of calcium supplementation on clinical fracture and bone structure: results of a 5-year, double-blind, placebo-controlled trial in elderly women. Arch Intern Med 166, 869-875.

55. Bolland MJ, Barber PA, Doughty RN, et al. (2008) Vascular events in healthy older women receiving calcium supplementation: randomised controlled trial. BMJ 88, 262.

56. Bolland MJ, Grey A, Avenell A, et al. (2011) Calcium supplements with or without vitamin D and risk of cardiovascular events: reanalysis of the Women's Health Initiative limited access dataset and meta-analysis. BMJ 342, d2040.

57. Lewis JR, Radavelli-Bagatini S, Rejnmark L, et al. (2015) The effects of calcium supplementation on verified coronary heart disease hospitalization and death in postmenopausal women: a collaborative meta-analysis of randomized controlled trials. J Bone Miner Res 30, 165-175.

58. Reid IR, Bolland MJ, Avenell A, et al. (2011) Cardiovascular effects of calcium supplementation. Osteoporos Int 22, 1649-1658.

59. Burckhardt P (2011) Potential negative cardiovascular effects of calcium supplements. Osteoporos Int 22, 1645-1647.

60. Rubin MR, Rundek T, McMahon DJ, et al. (2007) Carotid artery plaque thickness is associated with increased serum calcium levels: the Northern Manhattan study. Atherosclerosis 194, 426-432.

61. Foley RN, Collins AJ, Ishani A, et al. (2008) Calcium-phosphate levels and cardiovascular disease in community-dwelling adults: the Atherosclerosis Risk in Communities (ARIC) Study. Am Heart J 156, 556-563.

62. Bolland MJ, Wang TKM, van Pelt NC, et al. (2010) Abdominal aortic calcification on vertebral morphometry images predicts incident myocardial infarction. J Bone Miner Res 25, 505-512.

63. Reid IR, Schooler BA, Hannan SF, et al. (1986) The acute biochemical effects of four proprietary calcium preparations. Aust N Z J Med 16, 193-197.

64. Burt MG, Mangelsdorf BL, Petersons CJ, et al. (2013) Acute effect of calcium citrate on serum calcium and cardiovascular function. J Bone Miner Res 28, 412-418.

65. Reid IR, Bolland MJ \& Grey A (2010) Does calcium supplementation increase cardiovascular risk? Clin Endocrinol (Oxf) 73, 689-695.

66. Vlachopoulos C, Aznaouridis K \& Stefanadis C (2010) Prediction of cardiovascular events and all-cause mortality with arterial stiffness: a systematic review and meta-analysis. I Am Coll Cardiol 55, 1318-1327.

67. Mitchell GF, Hwang S-J, Vasan RS, et al. (2010) Arterial stiffness and cardiovascular events the Framingham Heart Study. Circulation 121, 505-511.

68. Crichton GE, Elias MF, Dore GA, et al. (2012) Relations between dairy food intake and arterial stiffness: pulse wave velocity and pulse pressure. Hypertension 59, 1044-1051.

69. Ivey KL, Hodgson JM, Kerr DA, et al. (2015) The effect of yoghurt and its probiotics on blood pressure and serum lipid profile; a randomised controlled trial. Nutr Metab Cardiovasc Dis 25, 46-51.

70. Recio-Rodriguez JI, Gomez-Marcos MA, Patino-Alonso MC, et al. (2014) Association between fat amount of dairy products with pulse wave velocity and carotid intima-media thickness in adults. Nutr J 13, 37.

71. Livingstone KM, Lovegrove JA, Cockcroft JR, et al. (2013) Does dairy food intake predict arterial stiffness and blood pressure in men?: evidence from the Caerphilly Prospective Study. Hypertension 61, 42-47. 
72. Yaron M, Roach V, Izkhakov E, et al. (2014) Effects of a typical acute oral calcium load on arterial properties and endothelial function in healthy subjects. Eur J Clin Nutr 68, 608-612.

73. Reid IR, Mason B, Horne A, et al. (2002) Effects of calcium supplementation on serum lipid concentrations in normal older women: a randomized controlled trial. Am J Med 112, 343-347.

74. Chai W, Cooney RV, Franke AA, et al. (2013) Effects of calcium and vitamin D supplementation on blood pressure and serum lipids and carotenoids: a randomized, double-blind, placebo-controlled, clinical trial. Ann Epidemiol 23, 564-570.

75. Karandish M, Shockravi S, Jalali MT, et al. (2009) Effect of calcium supplementation on lipid profile in overweight or obese Iranian women: a double-blind randomized clinical trial. Eur J Clin Nutr 63, 268-272.

76. Rajpathak SN, Xue X, Wassertheil-Smoller S, et al. (2010) Effect of $5 \mathrm{y}$ of calcium plus vitamin D supplementation on change in circulating lipids: results from the Women's Health Initiative. Am J Clin Nutr 91, 894-899.

77. Reid IR, Ames R, Mason B, et al. (2010) Effects of calcium supplementation on lipids, blood pressure, and body composition in healthy older men: a randomized controlled trial. Am J Clin Nutr 91, 131-139.

78. Lorenzen JK, Nielsen S, Holst JJ, et al. (2007) Effect of dairy calcium or supplementary calcium intake on postprandial fat metabolism, appetite, and subsequent energy intake. $\mathrm{Am} \mathrm{J}$ Clin Nutr 85, 678-687.

79. da Silva Ferreira T, Torres MR \& Sanjuliani AF (2013) Dietary calcium intake is associated with adiposity, metabolic profile, inflammatory state and blood pressure, but not with erythrocyte intracellular calcium and endothelial function in healthy pre-menopausal women. Br J Nutr 110, 1079-1088.

80. Rice BH, Cifelli CJ, Pikosky MA, et al. (2011) Dairy components and risk factors for cardiometabolic syndrome: recent evidence and opportunities for future research. $A d v$ Nutr 2, 396-407.

81. Xiao Q, Murphy RA, Houston DK, et al. (2013) Dietary and supplemental calcium intakes in relation to mortality from cardiovascular diseases in the NIH-AARP Diet and Health Study. JAMA Intern Med 173, 639-646.

82. Astrup A (2014) Yogurt and dairy product consumption to prevent cardiometabolic diseases: epidemiologic and experimental studies. Am J Clin Nutr 99, 1235s-1242s.

83. Ditscheid B, Keller S \& Jahreis G (2005) Cholesterol metabolism is affected by calcium phosphate supplementation in humans. J Nutr 135, 1678-1682.

84. Bostick RM, Fosdick L, Grandits GA, et al. (2000) Effect of calcium supplementation on serum cholesterol and blood pressure. A randomized, double-blind, placebo-controlled, clinical trial. Arch Fam Med 9, 31-38; discussion 39.

85. Fleischman AI, Yacowitz H, Hayton T, et al. (1966) Effects of dietary calcium upon lipid metabolism in mature male rats fed beef tallow. J Nutr 88, 255-260.

86. Lappe JM, Travers-Gustafson D, Davies KM, et al. (2007) Vitamin D and calcium supplementation reduces cancer risk: results of a randomized trial. Am J Clin Nutr $\mathbf{8 5}$, 1586-1591. 